\title{
OPTICAL PROPERTIES OF SEMILADDER POLYMER FOILS
}

\author{
B. JARZAzBeK ${ }^{a}$, T. ORLIK ${ }^{b}$, J. CIsowski ${ }^{a, b}$, E. SchaB-BALCERzAK ${ }^{a}$ \\ AND D. SEK ${ }^{a}$ \\ ${ }^{a}$ Centre of Polymer Chemistry, Polish Academy of Sciences \\ P.O. Box 20, 41-819 Zabrze, Poland \\ ${ }^{b}$ Institute of Physics, Silesian University of Technology \\ P.O. Box 221, 40-019 Katowice, Poland
}

\begin{abstract}
Optical transmission of amorphous polyimidazopyrrolone foils (pyrrons) were investigated in the spectral range $200-3300 \mathrm{~nm}$. The investigated materials exhibit a high transmission level (up to $85 \%$ ) in a wide range from about 500 to $2750 \mathrm{~nm}$. The short wavelength edge of transmission depends on the pyrron chemical structure being different for ether-, amide- and ester bridges. When analysing the experimental data, we applied the approach used for amorphous materials in order to determine the electronic structure parameters which may be related to the influence of polyimidazopyrrolone bridge structure and structural disorder on the optical properties and probable electronic transitions.
\end{abstract}

PACS numbers: 71.20.Rv, 78.40.Me

\section{Introduction}

Polyimidazopyrrolones belong to the group of semiladder polymers characterised by interesting properties such as high thermal stability and dielectric properties. Therefore such polymers have been investigated for the last thirty years $[1,2]$ finding a wide range of applications, for example as membranes to separate various gases [3]. One of limitations for versatile applications of ladder polymers is their poor solubility in organic solvents. Introducing to the single-chain segments of some flexible groups such as amide, ether and ester between ladder segments should improve the solubility, but may influence the thermal stability of such semiladder polymers. Due to very poor solubility, determination of the molecular weight of investigated polymers was difficult; only the values of the reduced viscosity have been obtained and presented in [4-6]. The amorphous character of the polyimidazopyrrolone foils structure has been proved by X-ray diffraction method [7] and also by differential scanning calorymetry (DSC) investigations. The process 
of synthesis and some other properties of polyimidazopyrrolones (pyrrons) have been described in detail in papers [4-6].

The aim of this work was to study the optical properties of polyimidazopyrrolone foils in visible and near infrared spectral range as well as the influence of the chain structure on the transmission spectra and position of the absorption edge. The results of these investigations are presented for the first time and we have not found similar considerations in the literature. To obtain the energy gap and other energies describing the electronic structure, we have applied the method typical of amorphous materials and then, on the base of these values, the dependence of possible electronic transitions and structural disorder on the pyrron structure have been considered.

\section{Experimental}

The investigated semiladder polymers were obtained using low temperature polycondensation carried out in dimethylacetamide (DMA) and then the process of thermal cyclization was applied. The foils were formed by evaporating the solvent and heating in vacuum, in the temperature range $50-280^{\circ} \mathrm{C}$. The general structure of the investigated polyimidazopyrrolones is shown in Fig. 1.

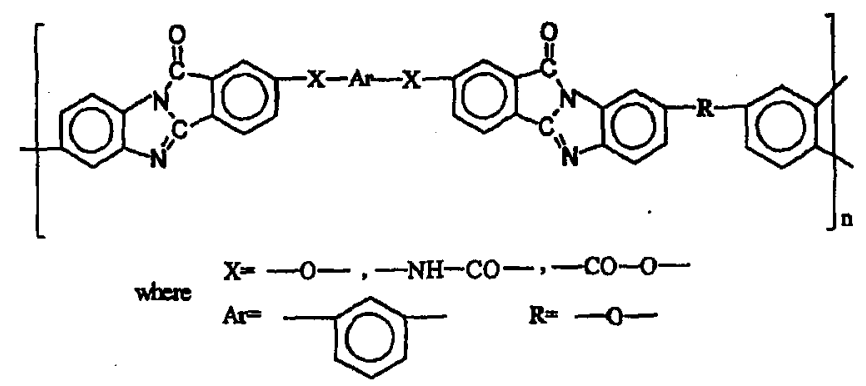

Fig. 1. Chemical structure of polyimidazopyrrolone with Ar, the central group, $R$, the tetramine group, and $\mathrm{X}$, the ether-, amide- and ester bridge in sequence.

In our optical measurements we used three types of foils with different bridges $(\mathrm{X})$ and the same central (Ar) and tetramine (R) groups as shown in Fig. 1. The thickness of polyesterimidazopyrrolone foil was $55 \mu \mathrm{m}$, being equal to $40 \mu \mathrm{m}$ for the foils with ether- and amide bridges.

The optical transmission measurements were performed at room temperature on BECKMAN Acta M-IV spectrophotometer within the $200-3300 \mathrm{~nm}$ range. A deuterium lamp was a source of ultraviolet, while tungsten lamps were used for visible and near infrared regions in this spectrophotometer.

\section{Results and discussion}

Typical transmission $(T)$ spectrum of investigated foils in the whole measurement range is shown in Fig. 2a. Sharp edges, one on the short wave side at 
about $500 \mathrm{~nm}$ and the other one on the long wave side at $2750 \mathrm{~nm}$, together with a high level of transmission, are characteristic of all types of foils. Such a good transparency (transmission up to $85 \%$ ) is caused by the absence of absorption, i.e. the lack of optical transitions in this wavelength range. The observed shape of the transmission curve indicates a possibility to use these polymers as optical filters in a wide spectral range. The short wavelength edge of transmission depends on the chemical structure of monomer, being different for ether-, amide- and ester bridges, while the edges on the long wave side are at the same position for all investigated foils. As can be seen in Fig. 2b, the light of shortest and longest wavelengths are transmitted by the foils with the ether- and ester bridges, respectively.
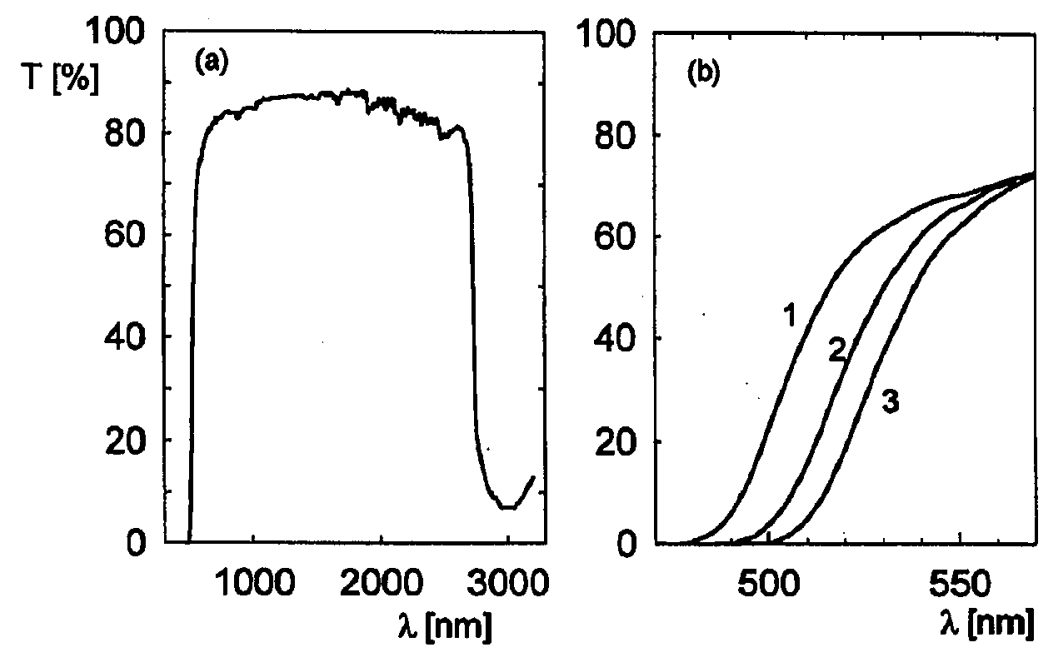

Fig. 2. (a) Typical overall transmission spectrum of polyimidazopyrrolone foil and (b) the short wave transmission edge of foils with the ether- (1), amide- (2) and ester (3) bridges.

In order to obtain the absorption coefficient $\alpha$ from the transmission data, the dependence $\alpha=(1 / d) \ln (1 / T)$, where $d$ is the foil thickness, was used. The shape of the absorption edges, presented for all foils in Fig. 3a as a function of energy $E$, is very similar to the absorption curve proposed by Tauc for a typical amorphous semiconductor [8-10]. The high level of absorption $\left(10^{4}-10^{5} \mathrm{~cm}^{-1}\right)$ is also typical of amorphous thin films [11, 12], indicating the presence of structural defects in the investigated foils. Each of the $\alpha(E)$ curves from Fig. 3a exhibits two exponential regions with different slopes. Higher energy exponential edges may be described by the Urbach relation $\alpha \sim \exp \left(E / E_{\mathrm{U}}\right)$ with the Urbach parameter $E_{\mathrm{U}}$, being similar for all the foils and equal to about $85 \mathrm{meV}$. Lower energy edges follow the same relation, with $E_{\mathrm{U}}$ replaced by the parameter $E_{\mathrm{T}}$ with its value different for the foils studied. For the photon energy $h \nu$ greater than the energy gap $E_{\mathrm{G}}$, the absorption edges for all investigated foils have been found to follow the Tauc power law $\alpha \sim\left(E-E_{\mathrm{G}}\right)^{2}$, as shown in Fig. 3b. This dependence, typical of amorphous semiconductors, is used to obtain the pseudogap for these type of 

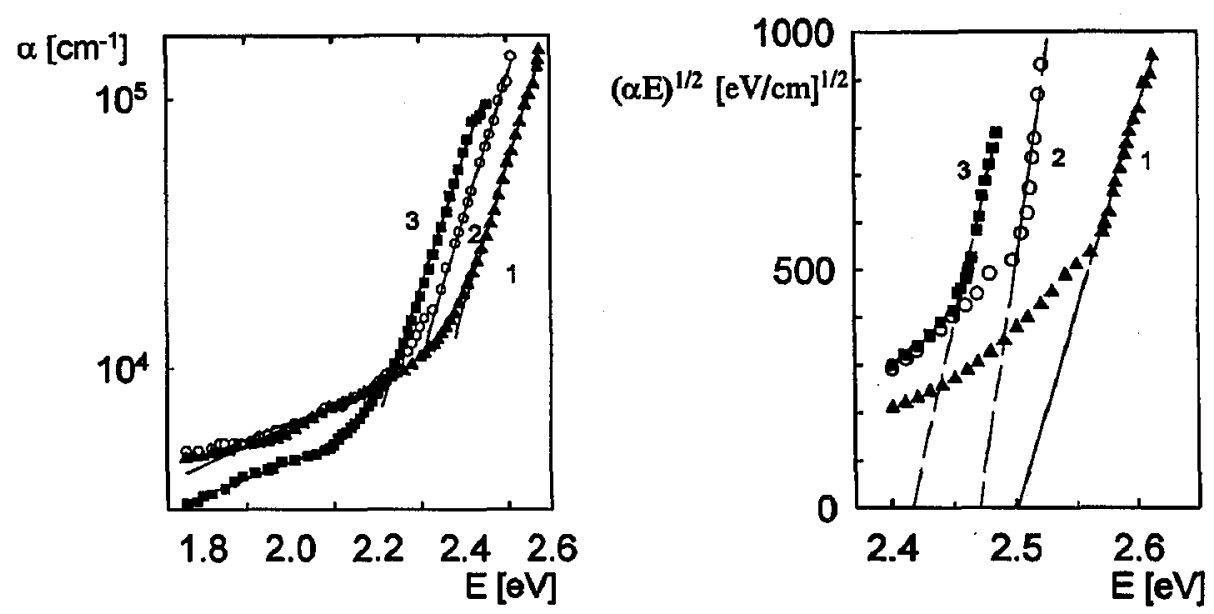

Fig. 3. (a) Absorption coefficient and (b) the Tauc dependence of the polyimidazopyrrolone foils with the ether- (1), amide- (2) and ester (3) bridges.

materials. The fact that the absorption data of pyrrons may be described by the Tauc relation confirms the amorphous character of investigated foils. Similarly as in other amorphous materials, the short range-order is present in this type of polymer, which is confirmed by existence of such distinct optical gaps. The energy gap values deduced from the $(\alpha E)^{1 / 2}$ vs. $E$ plots (Fig. $3 \mathrm{~b}$ ), change from $2.42 \mathrm{eV}$ for the foil with the ester bridge to $2.50 \mathrm{eV}$ for polyetherimidazopyrrolone foil. The values of optical parameters obtained for all investigated foils are gathered in Table.

\section{TABLE}

Optical parameters of investigated polyimidazopyrrolone foils.

\begin{tabular}{c|c|c|c|c}
\hline \hline Foil & Bridge $(\mathrm{X})$ & $E_{\mathrm{G}}^{\text {opt }}[\mathrm{eV}]$ & $E_{\mathrm{U}}[\mathrm{meV}]$ & $E_{\mathrm{T}}[\mathrm{meV}]$ \\
\hline 1 & ether & 2.50 & 83.8 & 533 \\
2 & amide & 2.47 & 86.2 & 808 \\
3 & ester & 2.42 & 88.7 & 628
\end{tabular}

Looking at the optical parameters presented in Table, one may observe that their values depend on the polyimidazopyrrolone bridge structure. The optical energy gaps of organic compounds in the near ultraviolet- and visible ranges are generally associated with the electronic transitions between the ground- and excited states, denoted as $\pi \rightarrow \pi^{*}$, i.e. between the bonding and antibonding states, while the parameters $E_{\mathrm{U}}$ and $E_{\mathrm{T}}$ describe the states inside the energy gap. The bridge structure of the investigated foils affects not only the values of $E_{\mathrm{G}}$ but also those of $E_{\mathrm{U}}$ and $E_{\mathrm{T}}$. The polyetherimidazopyrrolone foil has the largest energy gap and, simultaneously, the smallest values of $E_{\mathrm{U}}$ and $E_{\mathrm{T}}$. The structure of ether 
bridge seems to be the simplest one and hence its contribution to the structural disorder is smaller. Both amide- and ester bridges have the double bond between $\mathrm{O}$ and $\mathrm{C}$ atoms, which may cause to decrease energy gaps. A more complicated structures of amide- and ester bridges (with double bond) are probably responsible for smaller energy gaps and larger values of $E_{\mathrm{U}}$ and $E_{\mathrm{T}}$, indicating the existence of energy states near the highest occupied molecular orbital (HOMO or $\pi$ level) and the lowest unoccupied molecular orbital (LUMO or $\pi^{*}$ level) as well as the existence of energy states inside the gap.

\section{Conclusions}

The amorphous polyimidazopyrrolones foils seem to be very interesting materials as optical filters because of their high level of transmission (about $85 \%$ ) in the wide spectral range from about 500 to $2750 \mathrm{~nm}$. The short wave edge depends on the structure of polymer chain and behaves similarly as in amorphous inorganic semiconductors. This allowed us, for the first time, to determine the typical amorphous materials parameters, such as $E_{\mathrm{G}}^{\text {opt }}$ (the optical energy gap), $E_{\mathrm{U}}$ (the Urbach energy) and $E_{\mathrm{T}}$ from an analysis of the absorption curves of investigated foils. The bridge structure of polyimidazopyrrolone foils with the same centraland tetramine groups influences the values of all optical parameters. Our work shows usefulness of the UV-VIS spectroscopy which seems to be one of the simplest method for studying the electronic structure of polymer foils. In particular, in the case of polyimidazopyrrolones foils, it could be interesting to investigate the optical properties of such foils with different parts of the chain, for example with different central or tetramine groups.

\section{References}

[1] G. Arnold, J. Polym. Sci. Macromol. Rev. 14, 265 (1979).

[2] B. Nartissov, Macromol. Sci. Rev. Chem. C 11, 143 (1974).

[3] W.L. Zhou, X.S. Gao, F.C. Lou, J. Appl. Polym. Sci. 51, 855 (1994).

[4] D. Sęk, E. Schab-Balcerzak, E. Grabiec, Polymer 39, 7001 (1998).

[5] D. Sęk, E. Schab-Balcerzak, E. Grabiec, Polymer 40, 2419 (1999).

[6] D. Sęk, E. Schab-Balcerzak, E. Grabiec, Polymer 41, 49 (2000).

[7] A. Burian, private communication.

[8] E.A. Davis, N.F. Mott, Philos. Mag. 22, 903 (1970).

[9] J. Tauc, A. Menth, D.L. Wood, Phys. Rev. Lett. 25, 749 (1970).

[10] J. Tauc, A. Menth, J. Non-Cryst. Solids 8-10, 569 (1971).

[11] B. Jarząbek, J. Weszka, A. Burian, G. Pocztowski, Thin Solid Films 279, 204 (1996).

[12] A. Michalewicz, B. Jarząbek, J. Cisowski, J. Jurusik, A.M. Burian, J. Weszka, Electron Technol. 31, 411 (1998). 